\title{
Distal Middle Cerebral Artery Fusiform Aneurysm Associated with Systemic Lupus Erythematosus
}

\author{
Yu-Hsuan Lee ${ }^{2,4}$, Shun-Tai Yang ${ }^{1-4 *}$ \\ ${ }^{1}$ Graduate Institute of Medical Sciences, College of Medicine, Taipei Medical University, Taipei, Taiwan \\ ${ }^{2}$ Department of Neurosurgery, Shuang Ho Hospital, Taipei Medical University, Taipei, Taiwan \\ ${ }^{3}$ Department of Surgery, School of Medicine, College of Medicine, Taipei Medical University, Taipei, Taiwan \\ ${ }^{4}$ Taipei Neuroscience Institute, Taipei Medical University, Taipei, Taiwan
}

\section{Background}

The main etiology of spontaneous subarachnoid haemorrhage (SAH) is intracranial aneurysm. The patients often have risk factors associated with aneurysm formation such as hypertension, intracranial arteriovenous malformation and intracranial dural arteriovenous fistula. If the patients with aneurysms have no evident etiology, other uncommon etiologies such as vasculitis, infective endocarditis, and collagen vascular diseases should be surveyed especially when the aneurysm is located outside the circle of Willis in young patients. Herein, we presented a rare case with intracranial fusiform aneurysm located in distal middle cerebral artery (MCA). Interestingly, the patient was also diagnosed with systemic lupus erythema (SLE) in the absence of systemic activity. The association between this rare aneurysm and SLE was well discussed.

\section{Case report}

A 33-year-old female was found unconsciousness and sent to our hospital. Initial computed tomography $\mathrm{CT}$ ) scan revealed diffuse $\mathrm{SAH}$ (Figure 1A). Further angiography demonstrated a $1.2 \mathrm{~cm}$ fusiform aneurysm with irregularity of distal left MCA (Figure 1B). Tracing the past history of this patient, there was no evident risk factors associated with such an aneurysm formation. Between clipping reconstruction and trapping with cerebral revascularization, we chose the latter due to complex aneurysm morphology and unclear etiology of aneurysm formation. We performed a standard pterional approach, a fusiform aneurysm located at upper trunk of MCA was found. As torturousity and irregularity of the aneurysm made direct aneurysm clipping difficult and unsecure, a left superficial temporal artery to MCA bypass and trapping of lesioned parent artery were done to secure the ruptured fusiform aneurysm and preserve distal left MCA perfusion. The patient got uneventful recovery after surgical procedures. Postoperative CT angiography revealed patent anastomosis with preserved perfusion in the MCA territory (Figure 1C). The aneurysm wall was taken for pathological examination.

Further laboratory test indicated anti-nuclear antibody was1:1280X with a typical speckled pattern. Rheumatic factors Anti-Ro and Anti-La moderately elevated to $149.61 \mathrm{IU} / \mathrm{ml}$ and $386.84 \mathrm{IU} / \mathrm{ml}$ respectively. She developed malar rash outbreak 2 weeks after the surgery. Skin biopsy revealed picture of interface dermatitis with vacuolar degeneration of the basal layer of the epidermis. Also, it shows moderate C3 antibody marker granular deposition in the dermoepidermal junction and linear deposition in the basement membrane of hair follicles and sweat glands.
She was later diagnosed with SLE. On pathology, the aneurysm wall revealed granulation with fibrinous exudate and necrotic inflammation debris (Figure 1D), which are also compatible with pathological change in SLE.
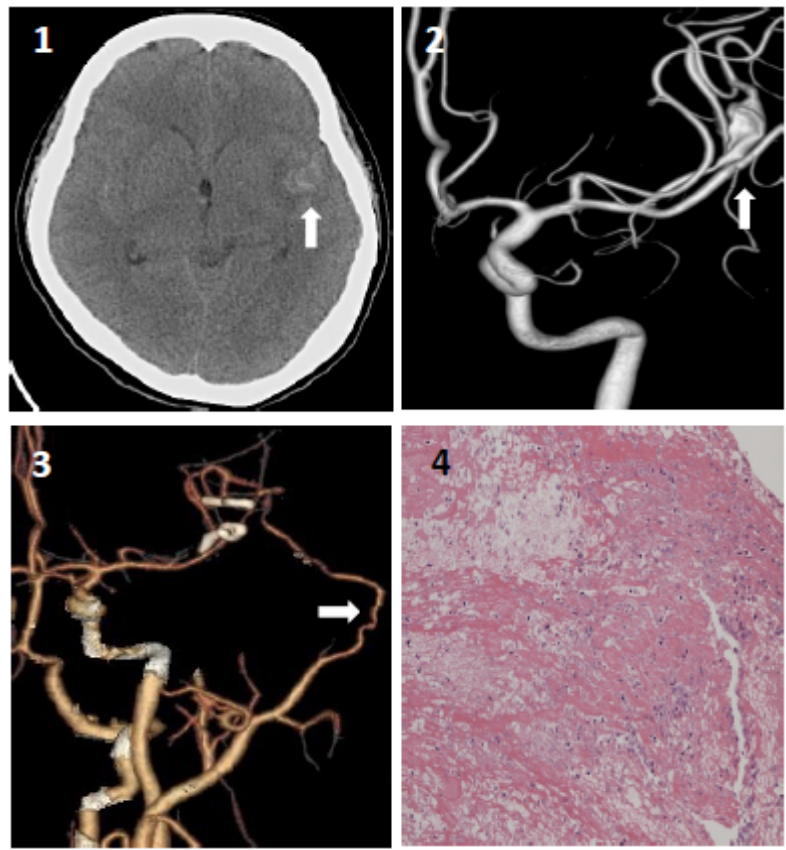

Figure 1. (1A). Brain computed tomography scan showed diffuse SAH dominantly over left sylvian fissure (white arrow). (1B). Reconstruction of left internal carotid artery angiography showed fusiform aneurysm at the left distal middle carotid artery (white arrow). (1C). Reconstruction of computed tomography angiography shows patent bypass artery (white arrow) from superficial temporal artery to distal middle carotid artery. Two aneurysm clips were applied for aneurysm trapping. (1D). Pathological study for the segment of aneurysm wall demonstrates fibrinous exudate and necrotic debris with additional lymphocytes, histiocytes, a few plasma cells, and rare polymorphonuclear cells (hematoxylineosin and Luxol fast blue, 20X)

${ }^{\star}$ Correspondence to: Shun-Tai Yang, Department of Neurosurgery, Shuang Ho Hospital, Taipei Medical University, Taipei, Taiwan, Tel: 886-2-22490088; Fax: 8862-22480900; E-mail: yst0708@gmail.com

Key words: systemic lupus erythematosus, fusiform aneurysm, spontaneous subarachnoid hemorrhage

Received: May 05, 2021; Accepted: May 21, 2021; Published: May 24, 2021 
To our knowledge, this is the first report of a distal fusiform intracranial aneurysm as initial manifestation of SLE in the absence of systemic activity. After 8 years of follow-up, brain MRI shows stationary patent ramification of left MCA and postoperative bypass artery with symmetrically good bilateral cerebral perfusion.

\section{Discussion}

SLE is an inflammatory disease that affects multiple organs and characterized by systemic involvement with heterogeneity of clinical features. Previous studies have suggested that 3-20\% of patients with SLE have cerebrovascular complications during the course of the diseases including ischemic stroke, transient ischemic attack, and less frequently hemorrhagic stroke and central venous thrombosis $[1,2]$. In a cross-sectional study reported $6.6 \%$ of SLE patients have cerebrovascular attack (CVA) during their disease course, small vessels thrombosis is the most common CVA subtype [3]. Vasculitis is one of the causes of CVA events. Vasculitis of peripheral artery may be noticed in as many as $56 \%$ of SLE patients throughout their life [4], but cerebral vasculitis is a rare manifestation, occurring in less than $7 \%$ of neuropsychiatric lupus and may occur without evidence of systemic disease activity [5]. For that reason, cerebral vasculitis is often over neglected in SLE patients. Vasculitis of cerebral vessels in SLE can lead to unstable endothelium which correlates with aneurysm formation and growth. These changes may predispose them to higher incidence of aneurysm formation. SLE patients associated with intracranial aneurysm consequently bear a worse prognosis with higher incidence of SAH, increased mortality, and worse Hunt and Hess grades on presentation [6].

In our patient, she endured SAH caused by aneurysm ruptured arising from peripheral segments of cerebral arteries which is relatively uncommon in general population. Young female without evident precipitating factors implied us to evaluate the causes of the aneurysm particularly when the aneurysm presented at unusual location. The causal relations between cerebral aneurysm and SLE have not yet been fully elucidated, but cognition of unusual pattern of SAH in clinical practice should raise the suspicion of cerebral vasculitis in autoimmune diseases patients.

\section{Conclusion}

Young patients with intracranial aneurysms at uncommon locations or atypical fusiform characteristic should be considered for further survey of uncommon etiologies. Autoimmune disease should be taken into the differential diagnosis in such a young patient presenting with atypical fusiform aneurysm without evident precipitating factors of aneurysm formation. Aneurysm trapping combined with bypass surgery should be carefully managed since direct aneurysm clipping and trans-arterial coiling is inadequate for such a ruptured distal fusiform aneurysm.

\section{Source of support}

Nil.

\section{Conflict of interest}

\section{No.}

\section{References}

1. de Amorim LC, Maia FM, Rodrigues CEM (2017) Stroke in systemic lupus erythematosus and antiphospholipid syndrome: risk factors, clinical manifestations, neuroimaging, and treatment. Lupus 26: 529-536. [Crossref]

2. Hanly JG, Li Q, Su L, Urowitz MB, Gordon C, et al. (2018) Cerebrovascular Events in Systemic Lupus Erythematosus: Results From an International Inception Cohort Study. Arthritis Care Res (Hoboken) 70: 1478-1487. [Crossref]

3. Saadatnia M, Sayed-Bonakdar Z, Mohammad-Sharifi G, Sarrami AH (2014) Prevalence and Prognosis of Cerebrovascular Accidents and its Subtypes Among Patients with Systemic Lupus Erythematosus in Isfahan, Iran: A Hospital Clinic-based Study. Int $J$ Prev Med 5: 123-126. [Crossref]

4. Radic M, Kaliterna DM, Radic J (2013) Vascular manifestations of systemic lupus erythematosis. Neth J Med 71: 10-16. [Crossref]

5. Everett CM, Graves TD, Lad S, Jager HR, Thom M, et al. (2008) Aggressive CNS lupus vasculitis in the absence of systemic disease activity. Rheumatology (Oxford) 47: 107-109. [Crossref]

6. Carvallo JFB, Brito CC, Estanol B, Ramos GSG (2007) Subarachnoid hemorrhage as a complication of systemic lupus erythematosus. Cerebrovasc Dis 24: 301-304. [Crossref]

Copyright: (C2021 Lee Y. This is an open-access article distributed under the terms of the Creative Commons Attribution License, which permits unrestricted use, distribution, and reproduction in any medium, provided the original author and source are credited. 